\section{The clinical significance of changes in red blood cell distribution width in patients with community-acquired pneumonia}

\author{
Sang-Min Lee, Jae Hyuk Lee, Kyuseok Kim , You Hwan Jo, \\ Jungyoup Lee, Joonghee Kim, Ji Eun Hwang, Young Sang Ko, \\ Chulmin Ha, Sujin Jang, Hyunmi Park
}

Department of Emergency Medicine, Seoul National University Bundang Hospital, Seongnam, Korea

Objective Red cell distribution width (RDW) is associated with mortality in patients with community-acquired pneumonia (CAP). However, little is known about the effect of changes in RDW during treatment on mortality. Thus, the objective of this study was to evaluate the association between RDW changes and mortality in hospitalized patients with CAP.

Methods Retrospective analyses were performed using medical records of patients hospitalized for CAP from April 2008 to February 2014. The abstracted laboratory values included RDW (from days one to four), clinical variables, and pneumonia severity index (PSI) scores. The $\triangle R D W_{n-1}$ was defined as the change in $\mathrm{RDW}$ calculated as: $\left(\mathrm{RDW}_{\text {day } 1}-\mathrm{RDW}_{\text {day-n }}\right) / \mathrm{RDW}_{\text {day } 1 \times 100}(\%)$, where 'day $\mathrm{n}$ ' refers to hospital day.

Results During the study period, a total of 1,069 patients were hospitalized for CAP. The 30-day mortality was 100/1,069 (9.4\%). The median RDW at baseline was 14.1\% (range, 11.1 to 30.2) and differed significantly between survivors and non-survivors $(P<0.05)$. There were 470 patients with available serial RDW data (30-day mortality 58/470 [12.3\%]). Of those, age, PSI score, blood urea nitrogen level, total protein concentration, albumin level, RDW at day 1 , and the $\triangle R D W_{4-1}$ differed significantly between survivors and non-survivors. Multivariate Cox regression analysis showed that the significance of the relationship between $\triangle R W_{4-1}$ and 30-day mortality risk remained after adjusting for age, PSI score, RDW at day 1, total protein concentration, and initial albumin level.

Conclusion RDW change from day 1 to day 4 was an independent predictor of mortality in patients with CAP.

Keywords Delta red cell distribution width; Red cell distribution width change; Community-acquired pneumonia

Capsule
Wummat is already known
$\begin{aligned} & \text { Red cell distribution width is an independent predictor of mortality in many } \\ & \text { diseases, including community-acquired pneumonia. }\end{aligned}$
$\begin{aligned} & \text { What is new in the current study } \\ & \text { In hospitalized patients with community-acquired pneumonia, change in red } \\ & \text { cell distribution width from baseline during treatment was an independent pre- } \\ & \text { dictor of mortality. }\end{aligned}$

elSSN: 2383-4625

Received: 22 April 2016

Revised: 16 June 2016

Accepted: 22 June 2016

Correspondence to: Jae Hyuk Lee Department of Emergency Medicine, Seoul National University Bundang Hospital, 82 Gumi-ro 173beon-gil, Bundang-gu, Seongnam 13620, Korea E-mail: hyukmd@gmail.com

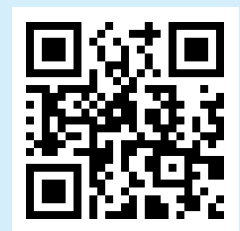

How to cite this article:

Lee SM, Lee JH, Kim K, Jo YH, Lee J, Kim J, Hwang JE, Ko YS, Ha C, Jang S, Park H. The clinical significance of changes in red blood cell distribution width in patients with community-acquired pneumonia. Clin Exp Emerg Med 2016;3(3):139-147.

This is an Open Access article distributed under the terms of the Creative Commons Attribution Non-Commercial License (http:// creativecommons.org/licenses/by-nc/4.0/). 


\section{INTRODUCTION}

Red cell distribution width (RDW) is the coefficient of variation of circulating red blood cells. This measure reflects the heterogeneity of the red cell population and has been used in diagnosing certain anemias, especially those that result from iron, vitamin B12, or folate deficiencies. ${ }^{1,2}$

RDW has recently been associated with outcomes of community-acquired pneumonia (CAP). ${ }^{3}$ CAP is the leading infectious cause of death worldwide and prognostication is an important part of its management. ${ }^{4,5}$ Inflammatory and oxidative stress caused by infection has been suggested as a mechanism for the association between RDW and infectious disease. ${ }^{6,7}$ Similarly, RDW reportedly showed a strong, graded association with inflammatory biomarkers in general outpatient populations. ${ }^{8}$ However, the relationship between change of RDW during treatment and outcome has not yet been identified, especially in patients with CAP. Increased RDW from baseline has recently been shown to predict mortality in patients with severe sepsis or septic shock. ${ }^{9}$ Thus, this study investigated the association between change in RDW during hospital admission and mortality in patients with CAP.

The hypothesis of this study is that the change of RDW during initial treatment is associated with mortality in hospitalized patients with CAP.

\section{METHODS}

\section{Study design and setting}

A retrospective analysis of a prospectively registered database of all consecutive patients with CAP was performed in a 1,200-bed tertiary academic hospital with an annual emergency department (ED) census of 85,000. The institutional review board of our institute approved the study and granted a waiver of informed consent.

\section{Participant selection}

Retrospective analyses were performed on medical records of patients hospitalized for pneumonia from April 2008 to February 2014. Eligible patients were older than 18 years of age and were diagnosed with CAP. CAP was defined as the presence of pulmonary infiltrate on chest radiographs and symptoms consistent with pneumonia, including cough, dyspnea, fever, and/or pleuritic chest pain, which were not acquired in a hospital or nursing home. If pulmonary infiltrate was not observed in the initial chest radiograph, abnormal lung sounds on the initial physical examination and pulmonary infiltrate on a follow-up chest radiograph were considered to be equivalent. Attending physicians in the ED determined patient admission based on pneumonia severity index
(PSI) scores and other medical conditions. The exclusion criteria included patients younger than 18 years of age as well as those who were discharged before the fourth day, who died before the fourth day, had any loss of daily value of complete blood count from the day of $E D$ visit to the fourth day of hospitalization, were transferred from another hospital, had used antibiotics within 10 days or had an episode of pneumonia within 30 days, were discharged from a hospital within 10 days, had active pulmonary tuberculosis, had known human immunodeficiency virus positivity, or had chronically immunosuppressed conditions (use of immunosuppressants for solid organ transplantation, post splenectomy, receiving $\geq 10 \mathrm{mg} /$ day prednisolone or equivalent for $<30$ days, treatment with other immunosuppressant, or neutropenia $\left[<1.0 \times 10^{9} / \mathrm{L}\right.$ neutrophils]). In addition, patients who had been at nursing home or community residential facilities, and those who were assumed to have aspiration pneumonia from preceding aspiration events were also excluded.

\section{Data collection}

Baseline clinical information was obtained through structured patient or proxy interviews, bedside assessments, and structured medical record reviews. Age; sex; comorbidities (including chronic obstructive pulmonary disease; diabetes mellitus; neoplasm; heart failure; and hepatic, renal, and central nervous system diseases); vital signs (blood pressure, heart rate, respiratory rate, and body temperature); and initial blood laboratory data including leukocyte count, hemoglobin, hematocrit, mean corpuscular volume, mean corpuscular hemoglobin, RDW, platelet count, blood glucose, serum creatinine, blood urea nitrogen, albumin, total cholesterol, total protein, prothrombin time (international normalized ratio), activated partial thromboplastin time, and C-reactive protein (CRP) levels; and daily RDW data were obtained.

\section{Methods of measurement}

RDW was measured using an automated hematology analyzer (Sysmex XE-2100; Sysmex, Kobe, Japan) during the first to fourth day of hospitalization and was reported as a part of the complete blood count results. $\triangle R D W_{n-1}(\%)$ was defined as (RDW day1-RDWday-n) $/ \mathrm{RDW}_{\text {day } 1} \times 100(\%)$, and $\triangle \mathrm{RDW}_{2-1}, \Delta \mathrm{RDW}_{3-1}$, and $\triangle \mathrm{RDW}_{4-1}$ values were calculated. Thirty-day mortality was ascertained by examining medical records during the hospital stay and by telephone after discharge. This study was based on a CAP registry database. All patients diagnosed with CAP in the ED are enrolled in this registry. If patients had been enrolled in our registry, study nurses attempted to determine their disposition from the ED and their status one month after the initial ED visit, using medical records for patients who died during the hospital stay. The status of 
patients discharged alive or transferred to another facility was ascertained based on medical records from the outpatient department one month after the ED visit. If medical records did not exist beyond one month of the ED visit, patients or surrogates were contacted by telephone. A total of five attempts to contact patients were made within one week.

\section{Statistical analysis}

Data were expressed as means \pm standard deviations. Baseline characteristics were compared between survivors and non-survivors. Continuous variables were analyzed using Student's t-test or Mann-Whitney U-test; categorical variables were analyzed using chi-square test or Fisher's exact test. Multivariable logistic regression analysis was performed to identify independent risk factors associated with 30-day mortality. To identify the association between $\triangle \mathrm{RDW}_{\mathrm{n}-1}$ and 30-day mortality, Cox proportional hazard regression analysis was performed, adjusting for variables that differed significantly between survivors and non-survivors.

To test the effect of initial RDW and $\triangle \mathrm{RDW}$ on survival time, patients were categorized according to cutoff values of RDW at day 1 and $\triangle \mathrm{RDW}$ using the highest sensitivity and specificity values based on receiver operator curve analysis. Kaplan-Meier survival analysis was performed according to RDW at day 1 and

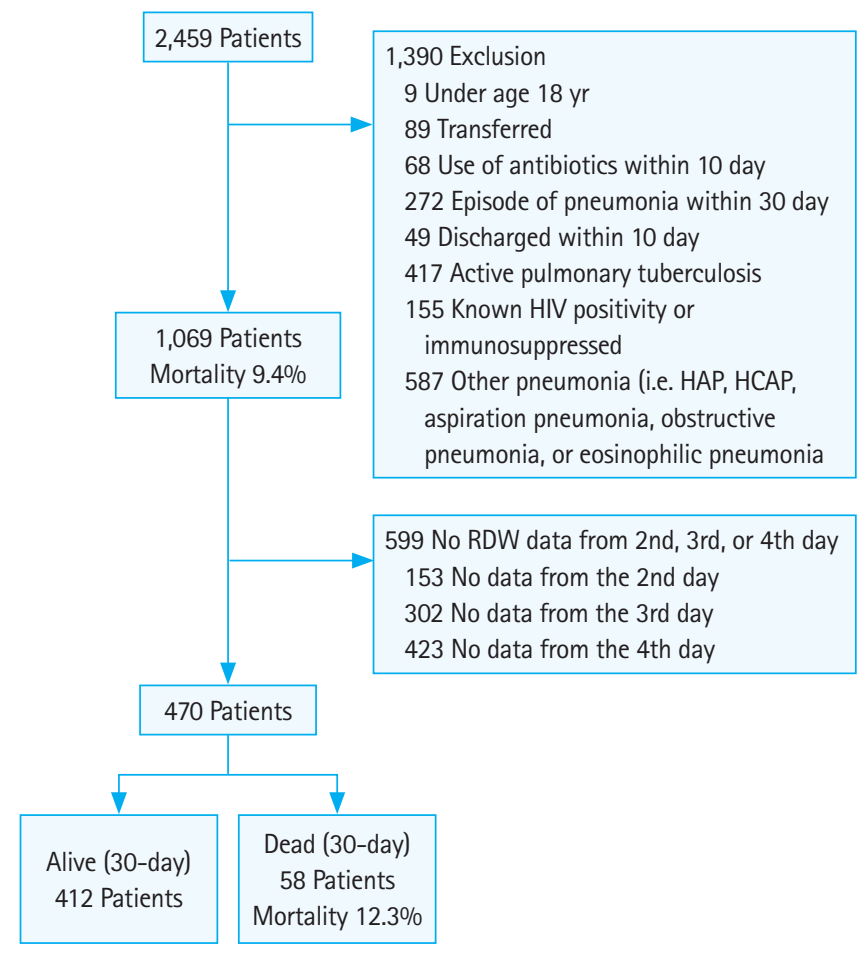

Fig. 1. The diagram describes selection process of study subjects. HIV, human immunodeficiency virus; HAP, hospital acquired pneumonia; HCAP, healthcare-acquired pneumonia; RDW, red cell distribution width. $\triangle \mathrm{RDW}$. All statistical analyses were performed using STATA/MP ver. 13.1 (StataCorp LP, College Station, TX, USA) and $\mathrm{P}<0.05$ was considered statistically significant.

\section{RESULTS}

\section{Baseline characteristics}

During the study period, 2,459 patients who visited the ED were diagnosed with pneumonia. Of those, 1,069 patients were hospitalized for CAP (Fig. 1). The 30-day mortality was 100/1,069 (9.4\%). The median RDW at baseline was $14.1 \%$ (range, 11.1 to 30.2 ) and

Table 1. Patient characteristics according to 30-day mortality

\begin{tabular}{|c|c|c|c|c|}
\hline Variable & $\begin{array}{l}\text { Total patients } \\
(n=470)\end{array}$ & $\begin{array}{c}\text { Alive } \\
(n=412)\end{array}$ & $\begin{array}{c}\text { Dead } \\
(n=58)\end{array}$ & P-value \\
\hline Age (yr) & $71.5 \pm 15.7$ & $70.9 \pm 16.0$ & $75.6 \pm 12.9$ & $<0.05$ \\
\hline Male sex (\%) & $323(68.7)$ & $283(68.7)$ & $40(69.0)$ & 0.97 \\
\hline \multicolumn{5}{|l|}{ Comorbidities } \\
\hline Heart failure & $13(2.8)$ & $12(2.9)$ & $1(1.7)$ & 1.00 \\
\hline Renal failure & $68(14.5)$ & $60(14.6)$ & $8(13.8)$ & 0.88 \\
\hline Liver disease & $27(5.7)$ & $25(6.1)$ & $2(3.4)$ & 0.56 \\
\hline COPD & 72 (15.3) & $66(16.0)$ & $6(10.3)$ & 0.18 \\
\hline Neoplasm & $100(21.3)$ & $84(20.4)$ & $16(27.6)$ & 0.21 \\
\hline Neurologic disease & $143(30.4)$ & $124(30.1)$ & $19(32.8)$ & 0.68 \\
\hline Diabetes mellitus & $155(33.0)$ & 139 (33.7) & $16(27.6)$ & 0.35 \\
\hline \multicolumn{5}{|l|}{ Laboratory findings } \\
\hline WBC & $13.3 \pm 8.2$ & $13.2 \pm 8.3$ & $13.4 \pm 7.3$ & 0.86 \\
\hline Hemoglobin level & $11.7 \pm 2.1$ & $11.8 \pm 2.1$ & $11.4 \pm 2.0$ & 0.18 \\
\hline RDW at day 1 & $14.8 \pm 1.9$ & $14.7 \pm 1.9$ & $15.6 \pm 2.1$ & $<0.05$ \\
\hline Hematocrit (\%) & $35.2 \pm 6.1$ & $35.3 \pm 6.1$ & $34.5 \pm 6.3$ & 0.40 \\
\hline MCV & $93.6 \pm 6.9$ & $93.5 \pm 6.7$ & $93.9 \pm 8.0$ & 0.71 \\
\hline $\mathrm{MCH}$ & $31.2 \pm 2.6$ & $31.2 \pm 2.6$ & $31.0 \pm 2.9$ & 0.53 \\
\hline Platelet $\left(\times 10^{3} / \mathrm{mm}^{3}\right)$ & $239.8 \pm 125.9$ & $238.7 \pm 127.0$ & $247.3 \pm 118.8$ & 0.63 \\
\hline Glucose (mg/dL) & $159.4 \pm 91.7$ & $158.0 \pm 93.0$ & $169.0 \pm 82.6$ & 0.39 \\
\hline Albumin (g/dL) & $3.3 \pm 0.6$ & $3.4 \pm 0.6$ & $3.1 \pm 0.6$ & $<0.05$ \\
\hline Cholesterol (g/dL) & $137.8 \pm 40.1$ & $137.0 \pm 39.4$ & $143.3 \pm 45.0$ & 0.26 \\
\hline BUN (mg/dL) & $28.9 \pm 21.2$ & $28.2 \pm 20.7$ & $34.3 \pm 24.1$ & $<0.05$ \\
\hline Total protein & $6.4 \pm 0.9$ & $6.4 \pm 0.8$ & $6.2 \pm 0.9$ & $<0.05$ \\
\hline Creatinine (mg/dL) & $1.5 \pm 1.6$ & $1.5 \pm 1.5$ & $1.8 \pm 1.8$ & 0.22 \\
\hline $\mathrm{Na}$ & $135.9 \pm 7.0$ & $136.0 \pm 6.9$ & $135.1 \pm 7.5$ & 0.35 \\
\hline K & $4.2 \pm 0.7$ & $4.2 \pm 0.7$ & $4.3 \pm 0.9$ & 0.43 \\
\hline $\mathrm{Cl}$ & $100.0 \pm 7.7$ & $100.2 \pm 7.7$ & $99.2 \pm 7.6$ & 0.38 \\
\hline PT (INR) & $1.3 \pm 1.2$ & $1.3 \pm 1.2$ & $1.3 \pm 0.6$ & 0.96 \\
\hline APT (sec) & $43.7 \pm 11.8$ & $43.9 \pm 12.3$ & $42.7 \pm 7.3$ & 0.47 \\
\hline CRP (mg/dL) & $14.2 \pm 9.3$ & $14.1 \pm 9.3$ & $14.9 \pm 8.8$ & 0.53 \\
\hline PSI score & $112.5 \pm 39.1$ & $109.5 \pm 38.9$ & $134.0 \pm 33.6$ & $<0.05$ \\
\hline PSI class & & & & $<0.05$ \\
\hline I, II & $61(13.0)$ & $59(14.3)$ & $2(3.4)$ & \\
\hline III & 73 (15.5) & 70 (17.0) & $3(5.2)$ & \\
\hline IV & $190(40.4)$ & $166(40.3)$ & $24(41.4)$ & \\
\hline V & $146(31.1)$ & $117(28.4)$ & $29(50.0)$ & \\
\hline
\end{tabular}

Values are presented as mean \pm standard deviation or number $(\%)$. COPD, chronic obstructive pulmonary disease; WBC, white blood cell; RDW, red blood cell distribution width; MCV, mean corpuscular volume; $\mathrm{MCH}$, mean corpuscular hemoglobin; BUN, blood urea nitrogen; PT, prothrombin time; INR, international normalized ratio; APT, activated partial thromboplastin time; CRP, Creactive protein; $\mathrm{PSI}$, pneumonia severity index. 
differed significantly between survivors (median RDW, 14.3\%; range, 12 to 25.5 ) and non-survivors (median RDW, 15.4\%; range, 12.6 to 24$)(P<0.05)$.

There were 470 patients with available serial RDW data (Fig. 1). The mean age was $71.5 \pm 15.7$ years, and $68.7 \%$ of patients were male. The overall 30-day mortality was 58/470 (12.3\%). Table 1 and Supplementary Table 1 show the demographic and clinical characteristics of the study population. Univariate analysis revealed that age; PSI scores; blood urea nitrogen, total protein, and albumin levels; and RDW at day 1 differed significantly between survivors and non-survivors.

\section{Association between $\triangle \mathrm{RDW}_{\mathrm{n}-1}$ and 30-day mortality}

Table 2 and Fig. 2 show the $\triangle R D W_{n-1}$ according to 30-day mortality. Of the $\triangle R D W_{n-1}, \Delta R D W_{4-1}$ differed significantly between survivors and non-survivors. To assess $\triangle R D W_{4-1}$ as an independent risk factor of 30-day mortality in patients with CAP, multivariate logistic regression analyses were performed after adjusting for variables that were significant in univariate analysis (Table 3). RDW at day $1, \triangle R W_{4-1}, P S I$ score, and albumin level were

Table 2. The 30-day mortality and its association with $\triangle \mathrm{RDW}$ of the second, third, and fourth day

\begin{tabular}{lcccc}
\hline Variable & $\begin{array}{c}\text { Total patients } \\
(\mathrm{n}=470)\end{array}$ & $\begin{array}{c}\text { Alive } \\
(\mathrm{n}=412)\end{array}$ & $\begin{array}{c}\text { Dead } \\
(\mathrm{n}=58)\end{array}$ & P-value \\
\hline$\Delta \mathrm{RDW}_{2-1}(\%)$ & $0.22 \pm 4.04$ & $0.22 \pm 4.11$ & $0.20 \pm 3.52$ & 0.96 \\
$\Delta \mathrm{RDW}_{3-1}(\%)$ & $0.12 \pm 4.65$ & $0.20 \pm 4.74$ & $-0.50 \pm 3.95$ & 0.28 \\
$\Delta \mathrm{RDW}_{4-1}(\%)$ & $0.60 \pm 4.72$ & $0.78 \pm 4.77$ & $-0.67 \pm 4.18$ & $<0.05$ \\
\hline
\end{tabular}

RDW, red cell distribution width.

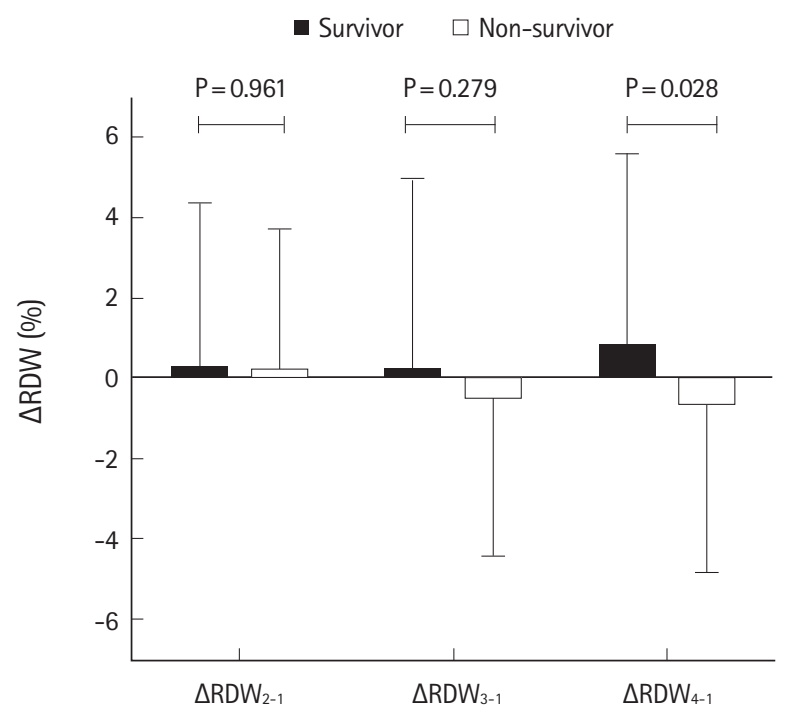

Fig. 2. Box graph of second, third, and fourth day $\triangle \mathrm{RDW}$ from day 1. RDW, red cell distribution width. identified as statistically significant variables. In Cox proportional hazard regression analysis, the significance of the relationship between $\triangle \mathrm{RDW}_{4-1}$ and 30-day mortality remained after adjusting for age, PSI score, RDW at day 1, and total protein and initial albumin levels (Table 4).

\section{Mortality according to initial RDW and $\triangle \mathrm{RDW}$}

RDW at day 1 and $\triangle R W_{4-1}$ were independently associated with 30-day mortality in patients with CAP. Additional analysis was performed to identify the effect of the RDW at day 1 and $\triangle R D W_{4-1}$ on survival time. The cutoff value was $14.8 \%$ for RDW at day 1 and $0.6 \%$ for $\triangle \mathrm{RDW}_{4-1}$ (Supplementary Fig. 1). Fig. 3 shows KaplanMeier survival curves according to RDW at day 1 and $\triangle$ RDW $_{4-1}$. Patients who had RDW $\leq 14.8 \%$ and $\triangle \mathrm{RDW}_{4-1} \geq 0.6 \%$ showed the highest survival time. Table 5 shows the stratified analysis of the effects of PSI class, initial RDW values, and $\triangle R W_{4-1}$ on mortality. When categorized into same PSI class, patients with initial RDW $\leq 14.8 \%$ showed a tendency towards lower mortality than those with RDW $>14.8 \%$. In addition, when categorized into the same PSI classes and initial RDW values, patients with $\triangle R D W_{4-1}$ $\geq 0.6 \%$ tended to have lower mortality rates than those with $\triangle \mathrm{RDW}_{4-1}<0.6 \%$.

Table 3. Multivariate logistic regression analysis for prediction of the 30-day mortality

\begin{tabular}{lcc}
\hline Variable & Odds ratio $(95 \% \mathrm{Cl})$ & P-value \\
\hline$\Delta$ RDW $_{4-1}$ & $0.00(0.00-0.50)$ & $<0.05$ \\
RDW at day 1 & $1.26(1.07-1.47)$ & $<0.05$ \\
PSI score & $1.02(1.00-1.03)$ & $<0.05$ \\
Age & $0.99(0.97-1.02)$ & 0.73 \\
Sex & $1.07(0.52-2.20)$ & 0.85 \\
BUN & $0.99(0.98-1.01)$ & 0.42 \\
Total protein & $1.08(0.68-1.73)$ & 0.74 \\
Albumin & $0.43(0.20-0.95)$ & $<0.05$ \\
\hline
\end{tabular}

$\mathrm{Cl}$, confidence interval; RDW, red blood cell distribution width; PSI, pneumonia severity index; BUN, blood urea nitrogen.

Table 4. Cox proportional hazard regression analysis for prediction of the 30-day mortality

\begin{tabular}{lcc}
\hline Variable & Hazard ratio $(95 \% \mathrm{Cl})$ & P-value \\
\hline$\Delta$ RDW $_{4-1}$ & $0.00(0.00-0.29)$ & $<0.05$ \\
RDW at day 1 & $1.21(1.07-1.37)$ & $<0.05$ \\
PSI score & $1.01(1.00-1.02)$ & $<0.05$ \\
Age & $0.99(0.97-1.02)$ & 0.69 \\
Sex & $1.14(0.60-2.15)$ & 0.69 \\
BUN & $1.00(0.98-1.01)$ & 0.55 \\
Total protein & $1.03(0.69-1.54)$ & 0.89 \\
Albumin & $0.48(0.24-0.96)$ & $<0.05$ \\
\hline
\end{tabular}

$\mathrm{Cl}$, confidence interval; RDW, red blood cell distribution width; PSI, pneumonia severity index; BUN, blood urea nitrogen. 
Table 5. Mortality according to initial RDW and $\triangle R W_{4-1}$

\begin{tabular}{|c|c|c|c|c|c|c|}
\hline \multirow{2}{*}{ PSI } & \multicolumn{2}{|c|}{ Initial RDW $\leq 14.8 \%$} & \multirow{2}{*}{ P-value } & \multicolumn{2}{|c|}{ Initial RDW > $14.8 \%$} & \multirow{2}{*}{ P-value } \\
\hline & $\Delta \mathrm{RDW}_{4-1} \geq 0.6 \%$ & $\Delta \mathrm{RDW}_{4-1}<0.6 \%$ & & $\Delta \mathrm{RDW}_{4-1} \geq 0.6 \%$ & $\Delta \mathrm{RDW}_{4-1}<0.6 \%$ & \\
\hline I, II & $0 / 25(0.0 \%)$ & $1 / 20(5.0 \%)$ & 0.44 & 0/8 (0.0\%) & $1 / 8(12.5 \%)$ & 1.00 \\
\hline III & $0 / 32(0.0 \%)$ & $1 / 20(5.0 \%)$ & 0.39 & $1 / 10(10.0 \%)$ & $1 / 11(9.1 \%)$ & 1.00 \\
\hline IV & $4 / 59(6.8 \%)$ & $5 / 58(8.6 \%)$ & 0.74 & $6 / 48(12.5 \%)$ & $9 / 25(36 \%)$ & $<0.05$ \\
\hline V & $3 / 23(13.0 \%)$ & $7 / 42(16.7 \%)$ & 1.00 & 10/58 (17.2\%) & 9/23 (39.1\%) & $<0.05$ \\
\hline
\end{tabular}

RDW, red blood cell distribution width; PSI, pneumonia severity index.

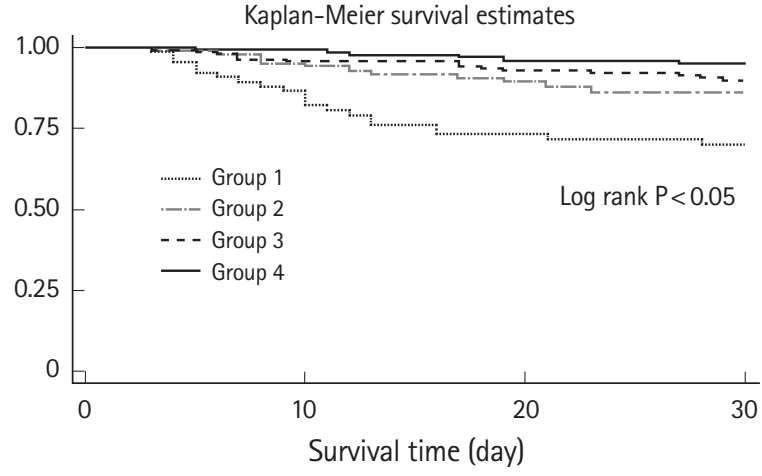

Fig. 3. Kaplan-Meier survival curves. Group 1: red cell distribution width (RDW) at day $1>14.8$ and $\triangle \mathrm{RDW}_{4-1}<0.6 \%$. Group 2 : RDW at day $1>14.8$ and $\triangle R D W_{4-1} \geq 0.6 \%$. Group 3 : RDW at day $1 \leq 14.8$ and $\triangle R D W_{4-1}<0.6 \%$. Group 4: RDW at day $1 \leq 14.8$ and $\triangle \mathrm{RDW}_{4-1} \geq 0.6 \%$.

\section{DISCUSSION}

This study demonstrates that RDW change during initial hospitalization is associated with 30-day mortality in hospitalized patients with CAP. RDW is associated with mortality in patients with many diseases, including cardiovascular disease, cancer, chronic lower respiratory disease, and cardiac arrest. ${ }^{10} \mathrm{RDW}$ is also associated with mortality in patients with infectious diseases like CAP and sepsis. ${ }^{3,11,12}$ The exact mechanism of the association between RDW and disease mortality has not yet been identified, although inflammation and oxidative stress have been suggested as potential mechanisms. In addition, RDW reportedly shows a strong, graded association with inflammatory markers in general populations ${ }^{8}$ and increased RDW from baseline values was significantly associated with mortality in patients with severe sepsis or septic shock. ${ }^{9}$ Furthermore, increasing changes in RDW are strongly correlated with inflammatory markers such as erythrocyte sedimentation rate (ESR) and CRP levels. ${ }^{13}$ Thus, changes in RDW during treatment may be associated with outcomes of inflammatory diseases and could be a marker of disease progression or resolution. In this study, change in RDW during initial treatment was independently associated with 30-day mortality in hospitalized patients with CAP. However, the association of RDW changes with inflammatory markers like ESR, CRP, procalcitonin, and inflammatory cytokine was not investigated in this study. Further study may be warranted.

Survival time was highest in patients with low RDW at baseline and decreased RDW during four days of hospitalization. This finding might be explained by resolution of inflammation and oxidative stress during initial treatment of CAP. Proinflammatory cytokines reportedly inhibit erythropoietin-induced erythrocyte maturation, ${ }^{7}$ systemic inflammation-influenced bone marrow function and iron metabolism, ${ }^{14,15}$ while oxidative stress shortened red blood cell survival and promoted release of premature red blood cells. ${ }^{6}$ Thus, during initial treatment, resolution of inflammatory and oxidative stress might decrease RDW, and baseline RDW values may reflect the initial inflammatory and oxidative stress.

In this study, RDW changes on the second and third days did not differ significantly between survivors and non-survivors. RDW decreased in survivors and increased in non-survivors during four days of hospitalization, and the change in RDW reached statistical significance on the fourth day. This observation is similar to other reports on the association between changes in RDW and mortality in patients with severe sepsis or septic shock. ${ }^{9}$ In those studies, 72-hour changes in RDW were associated with mortality in patients with severe sepsis or septic shock ${ }^{9}$ and 72 -hour RDW was a predictor of all-cause mortality in patients with gram-negative bacteremia. ${ }^{16}$ For this reason, we collected data from the first day to the fourth day of hospitalization. The number of cases was too small to include data from the fifth day $(n=296)$.

In this study population, albumin levels were an independent risk factor for 30-day mortality. However, CRP levels did not differ significantly between survivors and non-survivors. Previously, we reported that initial albumin and CRP were independent risk factors of mortality in patients with CAP. ${ }^{17}$ This discrepancy might be related to differences in patient populations. We included only those patients with daily RDW values available from the initial four days of hospitalization. In all patients hospitalized during the study period (1,069 patients), CRP levels differed significantly between survivors and non-survivors (data not shown; survivors $13.0 \pm 9.1 \mathrm{mg} / \mathrm{dL}$, non-survivors $15.7 \pm 8.9 \mathrm{mg} / \mathrm{dL})(\mathrm{P}<0.05)$. In 
addition, the CRP levels of non-survivors in this study were lower than those of all hospitalized patients. Thus, some patients who had severe disease and died before their fourth day of hospitalization might have been excluded. This may be a limitation of the current study.

This study has several limitations. First, it was conducted at a single institution and only included patients hospitalized via the $E D$. Therefore, it is difficult to generalize these findings to all CAP patients. Second, RDW is related to anemia or blood transfusion. However, data about transfusion, iron, vitamin B12, and folate levels was not collected. The median hemoglobin level in this study was $11.6 \mathrm{~g} / \mathrm{dL}$ (range, 5 to 17.9). Third, the initial RDW values may have influenced the decisions of the attending physicians regarding patient admission or discharge. However, in our hospital, most attending physicians determine the need for admission based on PSI scores and the general status of the patients, rather than RDW value. Fourth, as mentioned above, only those patients with serial RDW values over four days were included in this study. Thus, patients hospitalized for fewer than four days (mild cases or died on the third day) were excluded, which could result in a selection bias. Fifth, the changes of RDW were assumed to relate to systemic inflammation. However, the association of RDW changes with inflammatory markers, such as ESR, CRP, procalcitonin, and inflammatory cytokines was not analyzed. Furthermore, dynamic changes of biomarkers or clinical variables were neither evaluated nor adjusted. Further study is warranted. Sixth, RDW is associated with mortality in patients with cardiovascular and chronic obstructive pulmonary disease. In this study, 2.8\% and 15.3\% were heart failure and chronic obstructive pulmonary disease patients, respectively; however, no echocardiography or pulmonary function test data were available.

In summary, change in RDW from day 1 to day 4 was an independent predictor of mortality in patients with CAP. Follow-up, multi-center studies that include more patients are necessary. Further research is required to determine the precise mechanisms underlying the association between RDW and mortality; this study provides evidence for future investigations on RDW changes.

\section{CONFLICT OF INTEREST}

No potential conflict of interest relevant to this article was reported.

\section{ACKNOWLEDGMENTS}

This study was partly supported by SNUBH research fund (no. 022013-099).

\section{REFERENCES}

1. Schweiger DJ. Red cell distribution width in sickle cell anemia. Am J Med Technol 1981;47:231-3.

2. Bessman JD, Gilmer PR Jr, Gardner FH. Improved classification of anemias by MCV and RDW. Am J Clin Pathol 1983;80:322-6.

3. Lee JH, Chung $\mathrm{HJ}$, Kim K, et al. Red cell distribution width as a prognostic marker in patients with community-acquired pneumonia. Am J Emerg Med 2013;31:72-9.

4. Almirall J, Bolibar I, Vidal J, et al. Epidemiology of communityacquired pneumonia in adults: a population-based study. Eur Respir J 2000;15:757-63.

5. Armstrong GL, Conn LA, Pinner RW. Trends in infectious disease mortality in the United States during the 20th century. JAMA 1999;281:61-6.

6. Ghaffari S. Oxidative stress in the regulation of normal and neoplastic hematopoiesis. Antioxid Redox Signal 2008;10:192340.

7. Pierce $\mathrm{CN}$, Larson DF. Inflammatory cytokine inhibition of erythropoiesis in patients implanted with a mechanical circulatory assist device. Perfusion 2005;20:83-90.

8. Lippi G, Targher G, Montagnana M, Salvagno GL, Zoppini G, Guidi GC. Relation between red blood cell distribution width and inflammatory biomarkers in a large cohort of unselected outpatients. Arch Pathol Lab Med 2009;133:628-32.

9. Kim CH, Park JT, Kim E, et al. An increase in red blood cell distribution width from baseline predicts mortality in patients with severe sepsis or septic shock. Crit Care 2013;17:R282.

10. Perlstein TS, Weuve J, Pfeffer MA, Beckman JA. Red blood cell distribution width and mortality risk in a community-based prospective cohort. Arch Intern Med 2009;169:588-94.

11. Braun E, Domany E, Kenig Y, Mazor Y, Makhoul BF, Azzam ZS. Elevated red cell distribution width predicts poor outcome in young patients with community acquired pneumonia. Crit Care 2011;15:R194.

12. Mahmood NA, Mathew J, Kang B, DeBari VA, Khan MA. Broadening of the red blood cell distribution width is associated with increased severity of illness in patients with sepsis. Int J Crit IIIn Inj Sci 2014;4:278-82.

13. Arhan M, Onal IK, Tas A, et al. The role of red cell distribution width as a marker in inflammatory bowel disease. Turk J Med Sci 2011;41:227-34.

14. Chiari MM, Bagnoli R, De Luca PD, Monti M, Rampoldi E, Cunietti $\mathrm{E}$. Influence of acute inflammation on iron and nutritional status indexes in older inpatients. J Am Geriatr Soc 1995; 43:767-71.

15. Deswal A, Petersen NJ, Feldman AM, Young JB, White BG, Mann 
DL. Cytokines and cytokine receptors in advanced heart failure: an analysis of the cytokine database from the Vesnarinone trial (VEST). Circulation 2001;103:2055-9.

16. Ku NS, Kim HW, Oh HJ, et al. Red blood cell distribution width is an independent predictor of mortality in patients with gram- negative bacteremia. Shock 2012;38:123-7.

17. Lee JH, Kim J, Kim K, et al. Albumin and C-reactive protein have prognostic significance in patients with community-acquired pneumonia. J Crit Care 2011;26:287-94. 
Supplementary Table 1. Characteristics of included patients

\begin{tabular}{|c|c|c|c|}
\hline Variable & Total patients $(n=1,069)$ & Included patients $(n=470)$ & P-value \\
\hline Age (yr) & $71.1 \pm 15.6$ & $71.5 \pm 15.7$ & $<0.05$ \\
\hline Male (\%) & $726(67.9)$ & $323(68.7)$ & 0.31 \\
\hline Heart failure & $29(2.7)$ & $13(2.8)$ & 0.76 \\
\hline Renal failure & $141(13.2)$ & $68(14.5)$ & 0.93 \\
\hline Liver disease & $58(5.4)$ & $27(5.7)$ & 0.77 \\
\hline Neurologic disease & $310(29.0)$ & $143(30.4)$ & 0.12 \\
\hline Diabetes mellitus & $324(30.3)$ & $155(33.0)$ & 0.57 \\
\hline \multicolumn{4}{|l|}{ Laboratory findings } \\
\hline WBC & $12.8 \pm 7.1$ & $13.3 \pm 8.2$ & 0.67 \\
\hline Hemoglobin level & $12.0 \pm 2.0$ & $11.7 \pm 2.1$ & $<0.05$ \\
\hline RDW at day 1 & $14.5 \pm 1.9$ & $14.8 \pm 1.9$ & $<0.05$ \\
\hline Glucose (mg/dL) & $155.9 \pm 96.6$ & $159.4 \pm 91.7$ & 0.21 \\
\hline Albumin (g/dL) & $3.4 \pm 0.6$ & $3.3 \pm 0.6$ & $<0.05$ \\
\hline Cholesterol (g/dL) & $142.2 \pm 45.3$ & $137.8 \pm 40.1$ & 0.42 \\
\hline BUN (mg/dL) & $25.5 \pm 18.3$ & $28.9 \pm 21.2$ & $<0.05$ \\
\hline Total protein & $6.5 \pm 0.8$ & $6.4 \pm 0.9$ & $<0.05$ \\
\hline Creatinine (mg/dL) & $1.4 \pm 1.5$ & $1.5 \pm 1.6$ & 0.09 \\
\hline $\mathrm{Na}$ & $135.7 \pm 6.1$ & $135.9 \pm 7.0$ & 0.63 \\
\hline $\mathrm{K}$ & $4.2 \pm 0.7$ & $4.2 \pm 0.7$ & $<0.05$ \\
\hline $\mathrm{Cl}$ & $99.8 \pm 6.7$ & $100.0 \pm 7.7$ & 0.54 \\
\hline PT (INR) & $1.3 \pm 0.9$ & $1.3 \pm 1.2$ & 0.06 \\
\hline APT (sec) & $43.0 \pm 11.8$ & $43.7 \pm 11.8$ & 0.32 \\
\hline $\mathrm{CRP}(\mathrm{mg} / \mathrm{dL})$ & $13.2 \pm 9.1$ & $14.2 \pm 9.3$ & $<0.05$ \\
\hline
\end{tabular}

Values are presented as mean \pm standard deviation or number (\%).

COPD, chronic obstructive pulmonary disease; WBC, white blood cell; RDW, red blood cell distribution width; MCV, mean corpuscular volume; $\mathrm{MCH}$, mean corpuscular hemoglobin; BUN, blood urea nitrogen; PT, prothrombin time; INR, international normalized ratio; APT, activated partial thromboplastin time; CRP, C-reactive protein; PSI, pneumonia severity index. 


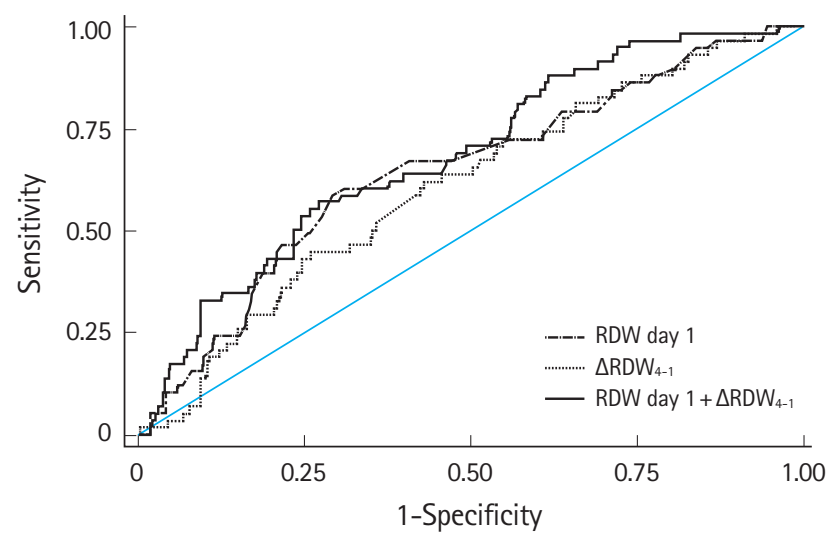

\begin{tabular}{lcc}
\hline Variable & AUC $(95 \% \mathrm{Cl})$ & P-value \\
\hline RDW day 1 & $0.64(0.57-0.72)$ & Reference \\
$\Delta$ RDW $_{4-1}$ & $0.61(0.53-0.68)$ & 0.13 \\
RDW day $1+\Delta$ RDW $_{4-1}$ & $0.68(0.61-0.75)$ & 0.07 \\
\hline
\end{tabular}

Supplementary Fig. 1. Receiver operating characteristics curve according to initial red cell distribution width (RDW) and $\triangle R D W_{4-1}$. AUC, area under the receiver operating characteristics curve; $\mathrm{Cl}$, confidence interval. 